\title{
FORMACIÓN COLABORATIVA \\ EN INTERCULTURALIDAD DEL PROFESORADO DE EDUCACIÓN INFANTIL Y PRIMARIA
}

\section{Early Childhood and Primary Education Teachers' Collaborative Training in Interculturality}

Rosabel Roig-Vila ${ }^{1}$

rosabel.roig@gcloud.ua.es

María Encarnación Urrea-Solano ${ }^{1}$ mayra.urrea@ua.es

${ }^{1}$ Universidad de Alicante, Espańa

Fecha recepción: 07/12/2019

Fecha aprobación: 31/12/2019

\section{Resumen}

La interculturalización de las prácticas educativas representa, hoy en día, uno de los principales retos a los que se enfrenta la escuela del siglo XXI. Para ello, la mejora de las competencias interculturales del profesorado parece constituir un requisito prioritario, sobre todo cuando esta se lleva a cabo desde un auténtico enfoque colaborativo y participativo. Por ello, esta investigación pretende Conocer las opiniones y experiencias de formación intercultural colaborativa del profesorado novel. Para el logro de este objetivo, se contó con la participación de diez docentes noveles que desempeñan su labor en un centro de Atención Educativa Preferente de Educación Infantil y Primaria de la Región de Murcia (España). El estudio se llevó a cabo desde un enfoque metodológico mixto, valiéndose para ello del software SPSS 21 y AQUAD 7. Cabe destacar de los resultados obtenidos que, si bien el profesorado novel reconoce la potencialidad de la cultura colaborativa para el desarrollo profesional docente, el principio de colaboración no acaba de ser plenamente valorado como una estrategia para el aprendizaje, sino más bien como un recurso para el funcionamiento institucional. La falta de oportunidades formales de capacitación colaborativa intercultural, tanto a nivel de centro, como en su formación inicial, es suplida por los docentes noveles mediante la colaboración con el resto de miembros del claustro. Los hallazgos del estudio contribuyen, en última instancia, a la propuesta de un modelo de aprendizaje profesional docente para poner en valor la diversidad presente en las aulas.

Palabras clave: aprendizaje colaborativo, Educación Infantil, Educación Primaria, interculturalidad, formación del profesorado, profesorado novel.

\section{Abstract}

The interculturalization of educational practices is, today, one of the main challenges facing the 21 st century school. To this end, the improvement of the intercultural competences of teachers seems to be a priority requirement, especially when it is carried out from a genuine collaborative and participatory approach. Based on this, this study was raised with the purpose of finding out the opinions and experiences of collaborative intercultural training of new teachers. To achieve this objective, ten novice teachers who worked in a Centre for Preferential Educational Care of Early Childhood and Primary Education in the Region of Murcia (Spain) participated. The study was carried out with a mixed methodological approach, using SPSS 21 and AQUAD 7 software. It is worth noting from the results obtained that, while novice teachers recognize the potential of collaborative culture for teaching professional development, the principle of collaboration has not been fully valued as a strategy for learning, but rather as a resource for institutional functioning. The lack of formal opportunities for intercultural collaborative training, both at the centre level and in their initial training, is filled by novice teachers through collaboration with the other members of the cloister. The findings of the study ultimately contribute to the proposal of a model of professional learning for teachers to value the diversity present in classrooms.

Keywords: collaborative learning, early Childhood, Primary education, interculturality, teacher training, novice teachers.

ISSN (impreso): 2636-2139

ISSN (en línea): 2636-2147

Sitio web: https://revistas.isfodosu.edu.do/recie 


\section{Introducción}

Desde hace algunas décadas, la práctica de la interculturalidad en los espacios escolares ha ganado un creciente reconocimiento y protagonismo en las agendas políticas e institucionales a nivel internacional. Como valor educativo, pero también como práctica encaminada a la mejora de la inclusión social, el fenómeno intercultural se ha convertido en un lugar común de los distintos programas y planes de capacitación docente. Así, diferentes estrategias, con resultados también variables, han sido diseñadas e implementadas con el fin de garantizar la competencia intercultural del profesorado, especialmente de quienes recién inician su andadura profesional.

Dentro de esta amplia gama de enfoques formativos, la literatura científica ha otorgado un papel relevante y privilegiado al aprendizaje colaborativo, dadas las potencialidades que presenta para lograr el compromiso docente con la diversidad étnico-cultural. A través de la interacción enriquecedora que promueve la cultura colaborativa en los centros escolares, el profesorado novel es capaz de reconstruir su mirada sobre el mundo y comprender la multiplicidad de sensibilidades e identidades que lo habitan. Ahora bien, aunque la incorporación del reto intercultural en la formación docente constituya una necesidad innegable y el principio colaborativo se perfila como el horizonte hacia donde caminar, también es preciso escuchar las voces del profesorado respecto a la validez y práctica de este tipo de iniciativas. Ante esta exigencia, el propósito del presente estudio fue identificar las creencias y experiencias de formación intercultural colaborativa del profesorado novel, que desempeña su labor en contextos caracterizados por la diversidad cultural del alumnado y del entorno. $\mathrm{El}$ análisis de los resultados permitirá, en última instancia, el diseño y configuración de una propuesta para el aprendizaje profesional docente en y para la diversidad.

\section{Revisión de la literatura}

En el cambiante escenario en el que vivimos, caracterizado por la velocidad constante y la incertidum- bre, las instituciones educativas y el profesorado deben hacer frente a las nuevas demandas y exigencias que le plantea la sociedad (Means, 2019). En este contexto, uno de los principales retos se halla en la definición misma del modelo educativo, ya que la escuela fabril e industrial de la pasada centuria parece no acabar de encajar en la sociedad actual del conocimiento, del capital social y de la globalización (Chiappe et al., 2019). La velocidad de las comunicaciones y la reducción de las distancias, entre lo global y lo local, han permitido ampliar los horizontes culturales y apreciar la diversidad intercultural que representa a este, nuestro mundo (United Nations Educational, Scientific and Cultural Organization [UNESCO], 2015), conformado, en última instancia, por la pluralidad cultural y la construcción de identidades híbridas (Delgado-Algarra et al., 2019). Indudablemente, esta expansión del paisaje cultural también afecta a las instituciones educativas y al profesorado, quien debe disponer de las competencias, destrezas y habilidades necesarias para afrontar los desafíos que emergen de la interculturalidad (Morales et al., 2017).

Ciertamente, la escuela, como microcosmos social, acoge y comprehende la diversidad de valores y culturas presentes en el espacio mundo (Alviar-Martin \& Baildon, 2017). Distintos modos de pensar, de sentir y de ser se encuentran e interaccionan en el medio escolar. Ahora bien, la construcción de la conciencia intercultural no surge de manera espontánea, sino que se precisa de acciones y de prácticas que alienten el desarrollo de un diálogo creador, tolerante y respetuoso entre las distintas sensibilidades culturales (Ohi et al., 2019) e inclusivas (Arnaiz et al., 2019). Ante este menester, la perspectiva intercultural se postula como el marco más adecuado para favorecer la auténtica colaboración y complementariedad entre las distintas culturas (Leiva, 2017). De acuerdo con Leeman \& van Koeven (2019), la educación intercultural permite responder a la diversidad de culturas presentes en la sociedad, mediante la valoración de la heterogeneidad como recurso y valor educativo. Parte de la premisa de que lo significativo es la interacción y el diálogo más profundo entre la diversidad de formas de entender la realidad; a través 
de estos, se alcanza la construcción conjunta de un nuevo marco de relaciones, basadas, especialmente, en la equidad, el respeto, la solidaridad y la justicia social (Aguado-Odina et al., 2017). Así, la interculturalidad podría ser definida como el enfoque educativo que busca y favorece el desarrollo de todo el alumnado, no solo de las minorías culturales (Biasutti et al., 2019), mediante el fortalecimiento de la identidad cultural y el fomento de la capacidad para comprender y valorar, respetuosa y críticamente, la cultura propia y ajena.

En este escenario pluricultural, la capacitación intercultural del profesorado representa una demanda cada vez más evidente y urgente, especialmente en el caso del novel (Stahl et al., 2019). En este contexto, numerosos estudios han venido a destacar el choque con la realidad que suelen experimentar los nuevos docentes al inicio de su carrera (Kelchtermans, 2019; Voss \& Kunter, 2019). Los sentimientos de aislamiento, así como la falta de apoyo y de orientación, son algunas de las características que definen sus primeros pasos en el ámbito profesional. En lo que respecta a la interculturalidad, tal y como Piot, Kelchtermans y Ballet (2010) vinieron a demostrar, la diversidad étnico-cultural del alumnado puede convertirse para el profesorado principiante en un importante factor de presión añadida, sobre todo como consecuencia de la distancia lingüístico-cultural que separa al docente y sus estudiantes. Hyry-Beihammer et al. (2019), por su parte, afirman que el profesorado novel percibe la diversidad del aula como una cuestión problemática, ajena a su capacidad de actuación y ante la que se reconoce incapaz. Frente a este incierto panorama, parece quedar fuera de toda duda la necesidad de incidir en la capacitación intercultural del profesorado y, por ende, determinar el modelo más adecuado para garantizar una preparación de calidad.

En este contexto, son múltiples los autores que sugieren posibles fórmulas de aprendizaje intercultural para el profesorado. Savva (2017), por ejemplo, señala que las experiencias en entornos multiinstitucionales y flexibles mejoran considerablemente las competencias interculturales de los docentes, al estimular el cuestionamiento de los propios valores y de la identidad profesional. Por su parte, Symeou y Karagiorgi (2018) enfatizan la utilidad de las prácticas reflexivas sobre la otredad y la naturaleza humana, como estrategia para facilitar la comprensión, la concienciación y la sensibilización del profesorado respecto a la diversidad étnico-cultural en las aulas. Sales et al. (2017), en cambio, centran el foco de atención en la complementariedad entre el aprendizaje y la investigación, conformando redes colaborativas que permitan el fortalecimiento de la formación práctica. Así, la colaboración y la revitalización del conocimiento generado a través de la propia práctica parecen convertirse en rasgos distintivos del nuevo paradigma de formación docente, en general (Fernández-Díaz et al., 2019), y en el ámbito intercultural, en particular (Peñalva \& Leiva, 2019).

En efecto, tanto la formación del profesorado como las problemáticas de la interculturalidad, requieren de procesos de intercambio de forma dialógica y creativa (Aguaded-Ramírez et al., 2018), es decir, del diálogo como un instrumento de conocimiento que se despliega en la interacción para abordar los problemas surgidos desde la práctica (Escarbajal, 2010). De hecho, son múltiples los estudios que evidencian la potencialidad de las estrategias colaborativas de formación para incrementar la competencia intercultural del profesorado. Entre otros, cabe citar, por ejemplo, la experiencia analizada por Little et al. (2019) con un programa online (iPGCE) dirigido a profesionales de la educación que trabajan en entornos multiculturales. La reflexión y la escritura colaborativa sobre la propia práctica docente, como núcleo fundamental del curso, resultó especialmente eficaz para desarrollar la conciencia e identidad intercultural de los participantes. Otra iniciativa similar es INTERACT, una plataforma online destinada a la creación de una comunidad virtual para el desarrollo profesional del profesorado en materia de interculturalidad (Hajisoteriou et al., 2018). Los efectos de su implementación evidenciaron las posibilidades que el aprendizaje colaborativo y dialógico ofrecen para reconstruir la cultura compartida en una búsqueda común y constante de significado intercultural. Resultados concomitantes fueron los hallados 
por Chamberlin-Quinlisk (2010) con un grupo de 16 docentes en lengua extranjera. A través de la elaboración de autobiografías compartidas y la revisión crítica literaria en pequeños grupos, el profesorado pudo, no solo experimentar el potencial de la cultura colaborativa para su aprendizaje, sino también que se pensaran a sí mismos como practicantes de una comunidad intercultural e interdisciplinar. Ahora bien, tal y como ha puesto de manifiesto el reciente estudio de Zeng y Day (2019), la mera consideración de un enfoque de aprendizaje como colaborativo no implica, necesariamente, el aumento del capital social o la mejora de la capacitación docente, sino que es preciso atender también a las particularidades y dinámicas que son propias de cada contexto específico.

\section{Método}

Con el propósito de aprehender la riqueza de matices de la realidad objeto de estudio, se optó por emplear un enfoque metodológico mixto, capaz de aunar las fortalezas y potencialidades de los métodos cualitativo y cuantitativo (Ponce \& Pagán-Maldonado, 2015).

\subsection{Contexto y participantes}

La investigación se realizó en un centro público de Educación Infantil y Primaria (CEIP) de la Región de Murcia (Espańa), ubicado en una zona con una alta presencia de población gitana e inmigrante, en su mayoría subsaharianos y magrebíes. Barrio humilde y trabajador desde sus orígenes, el contexto en el que se localizaba el centro ha sido, tradicionalmente, lugar de residencia de personas con un escaso nivel socioeconómico y foco de realojo municipal para familias de etnia gitana. A finales de la década de los años 90, comenzó a recibir población de origen inmigrante, atraída por las posibilidades que ofrecía el crecimiento económico del país. Sin embargo, en la actualidad, la zona se caracteriza por la existencia de graves problemas de vivienda y conflictividad sociocultural, elevadas tasas de desempleo y precariedad laboral, importantes índices de economía sumergida, así como una escasa formación de la población residente (Pastor \& Torralba, 2015). Todas estas ca- racterísticas le han valido para ser considerado como uno de los barrios más desfavorecidos de las ciudades españolas (Ministerio de Fomento, 2011).

Fiel reflejo del entorno, la población estudiantil del CEIP (254 estudiantes) en el que se enmarcó el estudio, se caracteriza por una notoria diversidad sociocultural: hay una escasa minoría de alumnado autóctono (12.89\%) y un considerable porcentaje de estudiantes de diverso origen étnico-cultural (87.11\%). En el momento del estudio, este último grupo estaba integrado por un importante número de estudiantes magrebíes $(42.15 \%)$, seguido por quienes eran de origen subsahariano $(34.78 \%)$ y gitano (10.18\%). Dado este carácter pluricultural del centro, la Conserjería de Educación y Cultura de la Región de Murcia otorgó al CEIP, desde el año 2016, la categoría de "Centro de Atención Educativa Preferente" (CAEP). Este reconocimiento se concretaba en un marco de compensación, interculturalidad y convivencia, mediante el desarrollo de medidas de atención preferente (recursos extraordinarios y reducción de ratio), el incremento de las ayudas y apoyos (becas) y la creación de un aula de acogida, actuaciones con las que se aspiraba a ofrecer una educación de calidad al conjunto del alumnado.

En cuanto a la plantilla del centro, estaba conformada por 32 docentes, de los cuales diez decidieron participar voluntariamente en el estudio. Respecto a las características sociodemográficas de la muestra, el 90\% de los docentes contaba, en el momento del estudio, con edades comprendidas entre los 20-30 años, siendo notablemente superior la participación del sexo femenino (80\%) frente al masculino. Según la experiencia docente, la totalidad de la muestra osciló entre los uno y cinco años, por lo que se trataba de un grupo de participantes que podía proporcionar información relevante sobre las experiencias y opiniones del profesorado novel hacia la formación colaborativa intercultural.

\subsection{Instrumento}

Para la recogida de la información se utilizó una adaptación del cuestionario de Domínguez (2006), diseñado para analizar la formación del profesorado 
ante la interculturalidad. Concretamente, se empleó el bloque de las cuestiones referidas al desarrollo profesional colaborativo en interculturalidad. El instrumento resultante quedó integrado por nueve ítems tipo Likert con cinco puntos de respuesta, que oscilaban entre Uno (Totalmente en desacuerdo) y Cinco (Totalmente de acuerdo). En muestras de población espańola se ha encontrado para esta dimensión un nivel $a l p h a(\alpha)$ y un coeficiente estandarizado de .89 , respectivamente. Estas cuestiones fueron complementadas, a su vez, con dos preguntas de carácter abierto sobre las experiencias de formación colaborativa e interculturalidad que los docentes habían tenido a lo largo de su vida profesional, y las actuaciones que el CEIP había desarrollado para estimular la cultura colaborativa. Como datos adicionales, se solicitó a los participantes que informaran sobre algunas variables sociodemográficas, como el sexo, la edad y los años de experiencia docente.

\subsection{Procedimiento}

Tras la autorización preceptiva del equipo directivo del CEIP, los datos relativos a las dimensiones estudiadas fueron recogidos en el centro escolar en el que trabajan los docentes que participaron en la investigación. El estudio fue presentado a estos como una investigación para profundizar en el conocimiento sobre su desarrollo profesional en el ámbito intercultural, asegurándoles la confidencialidad en las respuestas, el uso exclusivo de la información para la investigación y el carácter voluntario de la participación. Los cuestionarios fueron aplicados por una de las integrantes del equipo de investigación en un único momento, aprovechando para ello el horario de comida en el centro. A los participantes, que contestaron de forma individual, anónima y sin límite de tiempo, se les recordó que era muy importante que lo hicieran con total sinceridad. El tiempo medio empleado por los docentes para la cumplimentación del instrumento osciló entre los siete y diez minutos.

El tratamiento de los datos se realizó atendiendo a la naturaleza mixta de la investigación. En primer lugar, se llevó a cabo un estudio descriptivo básico con la ayuda del software SPSS versión 21. En segunda instancia, y con la ayuda del programa de análisis cualitativo AQUAD 7 (Huber \& Gürtler, 2013), se ejecutó un análisis de contenido convencional y sumativo (Hsieh \& Shannon, 2005). Con el primero de ellos, la información fue clasificada en unidades de significado, integradas dentro de categorías con un significado más amplio. El proceso de codificación siguió las fases de transcripción, agrupación, categorización y abstracción (Elo \& Kyngäs, 2008). Como resultado, se conformó un mapa de categorías y códigos que fue validado por tres expertas en investigación educativa. Mediante el recuento de las frecuencias del segundo análisis, se identificaron aquellos aspectos que las voces de los participantes destacaron con más insistencia.

\section{Resultados}

La exposición de los hallazgos atiende al enfoque mixto del estudio. Con base en ello, en primera instancia se presentan aquellos resultados de carácter cuantitativo y, posteriormente, aquellos de corte cualitativo.

\subsection{Resultados cuantitativos}

Tal y como se aprecia en la Tabla 1, los participantes no parecieron mostrar un posicionamiento claro y definido hacia la valoración de la colaboración como principio rector de la formación del profesorado. De hecho, la mitad de los docentes manifestó una postura indecisa y ambivalente ante la adecuación del enfoque colaborativo como base para el desarrollo profesional (ítem 1). Solo una escasa minoría se posicionó a favor de este tipo de estrategias para el aprendizaje del profesorado. En contraposición, hubo incluso algunos docentes que consideraron como inadecuada la dimensión colaborativa para la mejora profesional. En efecto, la elevada desviación típica en este ítem refleja la dispersión de las puntuaciones otorgadas. A pesar de esta tendencia ambivalente y variada, los participantes parecieron valorar positivamente la potencialidad de la cultura colaborativa para la formación docente (ítem 2). De hecho, el 60\% reconoció sus beneficios e, incluso, un $20 \%$ de los sujetos llegaron a afirmar que se trata de un enfoque metodoló- 
gico con numerosas posibilidades para optimizar su formación. Pese a ello, hubo algunos participantes que se mostraron incrédulos o que llegaron también a desacreditar su posible validez como fórmula para el progreso del profesorado.

\section{Tabla 1. Opiniones respecto a la formación docente colaborativa}

\begin{tabular}{lccccccc}
\hline Ítems & $\mathbf{1}$ & $\mathbf{2}$ & $\mathbf{3}$ & $\mathbf{4}$ & $\mathbf{5}$ & $\mathbf{M}$ & DT \\
\hline $\begin{array}{l}\text { 1.La formación basada en el principio de colaboración es } \\
\text { adecuada para el profesorado. }\end{array}$ & $0 \%$ & $20 \%$ & $50 \%$ & $0 \%$ & $30 \%$ & 3.40 & 1.174 \\
\hline $\begin{array}{l}\text { 2.La colaboración es una línea potente y coherente para } \\
\text { desarrollar al profesorado. }\end{array}$ & $0 \%$ & $10 \%$ & $10 \%$ & $60 \%$ & $20 \%$ & 3.90 & .876 \\
\hline $\begin{array}{l}\text { 3.Las experiencias de cultura colaborativa y trabajo for- } \\
\text { mativo en equipo en las que he participado han sido sa- } \\
\text { tisfactorias. }\end{array}$ & $0 \%$ & $10 \%$ & $30 \%$ & $40 \%$ & $20 \%$ & 3.70 & .949 \\
\hline $\begin{array}{l}\text { 4.El profesorado comparte habitualmente el conjunto de } \\
\text { experiencias formativas y de trabajo en equipo. }\end{array}$ & $0 \%$ & $0 \%$ & $20 \%$ & $40 \%$ & $40 \%$ & 4.20 & 7.89 \\
\hline
\end{tabular}

Nota: Elaboración propia.

Con respecto al nivel de satisfacción hacia las experiencias de formación colaborativa en las que se habían tomado parte, la desviación típica obtenida parece indicar una gran disparidad de opiniones entre los participantes (ítem 3). Ahora bien, la mayoría de las respuestas se concentraron en las categorías de valoración positiva. En este sentido, el $40 \%$ de los docentes reconoció estar conforme con las acciones de formación colaborativa en las que había intervenido, mientras que un $20 \%$ manifestó un alto grado de satisfacción. Frente a ello, el 30\% se mostró indiferente e, incluso, el 10\% expresó su descontento hacia este tipo de iniciativas.
Un posicionamiento más claro se entrevió en cuanto al nivel de colaboración de las experiencias formativas (ítem 4). En este caso, los docentes manifestaron una incontestable predisposición a la hora de compartir las vivencias de aprendizaje y el trabajo con el resto de compañeros y compañeras. Así, el 40\% de la muestra señaló que el equipo docente del centro suele difundir sus conocimientos y destrezas entre los miembros del claustro. Similar porcentaje de participantes reconoció que esta era una práctica muy habitual en el colegio, frente a una minoría que se posicionó de manera vacilante y dubitativa ante este tipo de prácticas.

Tabla 2. Estrategias de formación colaborativa en interculturalidad en el CEIP

\begin{tabular}{lccccccc}
\hline Ítems & $\mathbf{1}$ & $\mathbf{2}$ & $\mathbf{3}$ & $\mathbf{4}$ & $\mathbf{5}$ & $\mathbf{M}$ & $\mathbf{D T}$ \\
\hline 5.Proyectos interculturales compartidos en el centro. & $10 \%$ & $10 \%$ & $20 \%$ & $60 \%$ & $0 \%$ & 3.30 & 1.059 \\
\hline 6.Trabajos en equipos con otros centros. & $10 \%$ & $30 \%$ & $20 \%$ & $20 \%$ & $20 \%$ & 3.10 & 1.370 \\
\hline 7.Organización flexible de los equipos docentes. & $0 \%$ & $0 \%$ & $10 \%$ & $60 \%$ & $30 \%$ & 4.20 & .632 \\
\hline 8.Análisis de problemas inciertos y complejos. & $20 \%$ & $10 \%$ & $30 \%$ & $20 \%$ & $20 \%$ & 3.10 & 1.449 \\
\hline 9.Integración interdisciplinar. & $0 \%$ & $10 \%$ & $60 \%$ & $20 \%$ & $10 \%$ & 3.30 & .823 \\
\hline
\end{tabular}

Nota: Elaboración propia. 
En lo concerniente a las estrategias de formación colaborativa en interculturalidad (Tabla 2), el profesorado novel subrayó, la organización flexible del equipo docente como aquella más empleada en el centro (ítem 7). Además de esta, también destacaron, aunque con una menor puntuación, los proyectos interculturales desarrollados dentro de la institución (ítem 5) y la integración interdisciplinar (ítem 9). En cambio, las actuaciones coordinadas con otros centros (ítem 6) y el análisis de problemas inciertos y complejos (ítem 8), fueron las actuaciones que obtuvieron medias más bajas, pese a que se produjo una notable disparidad en las respuestas.

\subsection{Resultados cualitativos}

La información que emergió de los discursos de los participantes se organizó en torno a dos categorías: (1) Oportunidades de aprendizaje y (2) Medidas ins- titucionales, mientras que la primera se refiere a las experiencias de formación colaborativa que el profesorado novel había tenido en materia de interculturalidad, la segunda engloba las medidas que el CEIP había implementado para impulsar la formación docente y la cultura colaborativa en el centro.

\subsubsection{Experiencias de aprendizaje}

La Tabla 3 recoge los resultados de las frecuencias absolutas (FA) y el porcentaje de estas (\%FA), siendo FA el número de veces que los participantes aludían a una unidad de significado y \%FA la relación de dicho código con el total de FA (FAx100/total FA). Además de estos datos, la exposición de los resultados cualitativos se apoya en una serie de narrativas, extraídas de las voces de los participantes, que viene a ilustrar el significado de cada uno de los códigos emergentes.

Tabla 3. Frecuencias absolutas de los códigos pertenecientes la Categoría 1

\begin{tabular}{llcc}
\hline Categoría & \multicolumn{1}{c}{ Códigos } & FA & \%FA \\
\hline \multirow{3}{*}{ 1. Oportunidades de aprendizaje } & 1.1. Ausencia de cursos & 8 & 61.54 \\
\cline { 2 - 4 } & 1.2. Aprendizaje colaborativo & 4 & 30.76 \\
\cline { 2 - 4 } & 1.3. Ensayo y error & 1 & 7.7 \\
\hline Totales & & 13 & 100 \\
\hline \multirow{2}{*}{ Nota: Elaboración propia. } & & &
\end{tabular}

En cuanto a las experiencias de formación colaborativa en el ámbito de la interculturalidad, el profesorado novel destacó, con especial insistencia y sin ambages, la ausencia de actividades regladas en el centro. Algunos relatos así lo evidencian ["En este centro no se ha llevado a cabo, al menos durante estos dos últimos años, ningún curso de formación basado en estos aspectos, DOC_02; Desde que yo estoy en el centro no se ha hecho ningún curso", DOC_10]. Además, se ha constatado que esta carencia de oportunidades no estaba limitada únicamente al colegio, sino que sus discursos dejaban entrever que a lo largo de su formación inicial tampoco habían tenido posibilidad de ampliar sus conocimientos en este ámbito ["La formación reglada respecto al trabajo en equipo e interculturalidad es bastante limitada” DOC_03].
Esta situación los lleva a demandar más oportunidades de aprendizaje sobre interculturalidad, dadas, sobre todo, las características del centro ["Desgraciadamente ninguna y la verdad es que sería muy necesario, sobre todo por la zona donde está el centro", DOC_09].

En esta crítica coyuntura, los participantes señalaban, de manera reiterada, que esta falta de formación era compensada por el intercambio de experiencias y la colaboración diaria entre todos los miembros del equipo docente ["Lo que hacemos es hablar e intercambiar las ideas que se nos van ocurriendo y que funcionan", DOC_10]. Sin embargo, tras este tipo de iniciativas no parecía existir una intencionalidad consciente de aprendizaje, sino, más bien, una ne- 
cesidad motivada por las peculiaridades del centro ["El trabajo en equipo es algo que se trabaja a diario, más aún en este centro en el que los agrupamientos de alumnos son flexibles, por lo que todo el claustro debe estar en comunicación continua al tener en el aula alumnos de varias clases", DOC_06]. De hecho, estas prácticas parecían convertirse en un requisito básico para el correcto funcionamiento de la institución ["Pero diariamente se trabaja siempre en el centro partiendo de la colaboración entre todos para ayudar al correcto funcionamiento tanto del centro, clases, como del alumnado", DOC_07].

Frente a ello, también hubo alusiones, aunque con una frecuencia anecdótica, al conocimiento derivado del quehacer pedagógico. Esta estrategia de formación en la acción era considerada, a su vez, como un elemento clave para la mejora y el desarrollo profesional ["Pero sinceramente el día a día va dándonos aquellos conocimientos que ayudan a un mejor desempeño de nuestras labores como docentes en el centro", DOC_02].

\subsubsection{Actuaciones institucionales para el estímu- lo de la formación docente y de la cultura colabo- rativa}

Respecto al apoyo institucional a la formación colaborativa, segunda de las categorías, el análisis de las voces de los participantes evidenció que este se desarrollaba, mediante la implementación de actuaciones de carácter externo e interno, (Tabla 4). Entre las primeras, los participantes destacaron, con notoria insistencia, la apertura de vías de comunicación y colaboración con las asociaciones comunitarias que intervienen en la zona ["Colaborar con las actividades que realizan las asociaciones que trabajan en el barrio”, DOC_10]. Junto a estas, también enfatizaron la actuación coordinada con las familias del alumnado, iniciativa considerada como un elemento complementario al trabajo que realizan con las organizaciones que operan en el entorno del centro ["Sobre todo el trabajo con las familias y las asociaciones del barrio", DOC_01]. El profesorado novel consideró que el desarrollo equilibrado de ambas estrategias permite ofrecer, en última instancia, una respuesta adecuada a las necesidades socioeducativas del alumnado ["En el centro se tiene muy claro que la colaboración entre todos, con las familias y con las asociaciones que trabajan en el barrio es esencial para que nuestro alumnado sea atendido como debe", DOC_07].

\section{Tabla 4. Frecuencias absolutas de los códigos per- tenecientes la Categoría 2}

\begin{tabular}{llcc}
\hline Categoría & \multicolumn{1}{c}{ Códigos } & FA & \%FA \\
\hline \multirow{2}{*}{$\begin{array}{l}\text { 2. Medidas insti- } \\
\text { tucionales }\end{array}$} & $\begin{array}{l}\text { 1.1. De carácter } \\
\text { externo }\end{array}$ & 8 & 61.54 \\
\cline { 2 - 4 } & $\begin{array}{l}\text { 1.2 De carácter } \\
\text { interno }\end{array}$ & 5 & 38.46 \\
\hline Totales & & 13 & 100 \\
\hline
\end{tabular}

Nota: Elaboración propia.

En contraposición a este tipo de prácticas, los docentes también aludieron, aunque con menor frecuencia, a las medidas que el centro desarrolla a nivel de claustro. En este ámbito, subrayaron, de manera reiterativa, la celebración de reuniones de planificación entre todos los integrantes del equipo docente ["Reuniones de tramo en las que ponemos en común aquellos aspectos y técnicas que nos han resultado útiles y adecuadas", DOC_05]. Estos espacios de coordinación eran aprovechados no solo para el intercambio de conocimientos, sino también para la resolución de conflictos y problemas, así como para la programación consensuada del proceso de enseñanza y aprendizaje ["Las decisiones son diarias, ya que se establecen semanalmente reuniones de tramo en las que se ponen en común las experiencias y se buscan soluciones conjuntas a los problemas o dificultades que hayan podido surgir, para evitar que vuelvan a suceder”, DOC_06]. También en esta línea, pero con una escasa incidencia, mencionaron la búsqueda y establecimiento de tiempos, por parte del equipo directivo, para promover el trabajo en equipo ["Se ha hecho un planning para que dispongamos de tiempo para el trabajo en equipo", DOC_04].

En suma, el análisis de los datos permite afirmar que, si bien el principio de colaboración no es plenamen- 
te reconocido como estrategia de formación en interculturalidad por el profesorado novel, sí que este valora su potencialidad para el aprendizaje docente. Parece, junto a ello, que las oportunidades formativas de carácter colaborativo para la atención a la diversidad étnico-cultural son más bien escasas y limitadas, por lo que el profesorado novel se vale del aprendizaje informal para mejorar su capacitación en esta área. Además, aunque los resultados del estudio denotan la existencia de una cultura colaborativa en el centro, esta apenas es empleada como una vía para la formación, sino más bien como un recurso para la organización y funcionamiento diario de la institución. De hecho, la mayor parte de las iniciativas que el centro lleva a cabo para estimular la formación colaborativa del equipo docente tiene lugar mediante el desarrollo de actuaciones coordinadas con el entorno.

\section{Discusión y conclusiones}

Reconociendo la pertinencia y adecuación de las metodologías colaborativas para la capacitación del profesorado en interculturalidad, este estudio se planteó con el objetivo de identificar y reconocer las creencias y experiencias formativas del profesorado novel para la atención a la diversidad étnico-cultural. De acuerdo con los resultados, la gran mayoría de los participantes parecieron mostrarse escépticos ante la idoneidad de la cultura colaborativa para la mejora docente. Esta escasa confianza podría venir motivada por la desilusión y el desencanto propio del principiante al inicio de su desempeño profesional. De acuerdo con Kelchtermans (2019), el profesorado novel se encuentra perdido y solo durante sus primeros años de ejercicio. El acceso a la carrera profesional supone un periodo crítico de desconcierto y de crisis identitaria, que podría estar afectando a la percepción del principio colaborativo (Voss \& Kunter, 2019). A ello se ha de añadir, además, la posible confusión entre el aprendizaje colaborativo y aquel que se basa en la mera cooperación entre distintos agentes (Peńalva \& Leiva, 2019). Mientras que el segundo se limita a la mera ejecución de la tarea, el primero implica el surgimiento del sentimiento de comunidad, así como el compromiso y la interdependencia entre todos sus participantes.

A pesar de esta falta de reconocimiento, los participantes fueron capaces de apreciar las oportunidades que presenta el enfoque colaborativo como línea potente y coherente para el desarrollo profesional del profesorado. Tal y como numerosos autores han puesto de manifiesto (Escarbajal, 2010; Ohi et al., 2019; Sales et al., 2017), el intercambio de ideas, de experiencias y de conocimientos se convierte en una estrategia de extraordinaria eficacia para mejorar la competencia intercultural docente. A través de esta, el profesorado novel es capaz de superar la inseguridad, el desconcierto y los temores que le plantea la respuesta a la diversidad étnico-cultural (Piot et al., 2010). De hecho, los participantes en el estudio mostraron un notable grado de satisfacción respecto a las experiencias de cultura colaborativa en las que habían tomado parte. Resultados concomitantes fueron los hallados por Hajisoteriou et al. (2018), cuando subrayan las vivencias positivas que el profesorado experimenta cuando se siente parte integrante de una comunidad de aprendizaje en interculturalidad. Sentir que tienen voz y que esta es escuchada y reconocida constituye no solo una valiosa oportunidad de aprendizaje, sino también un potente ejercicio de construcción identitaria y de empoderamiento profesional para los docentes (Chamberlin-Quinlisk, 2010; Prendes \& Román, 2017).

Por otra parte, los resultados del estudio evidenciaron que el conocimiento en interculturalidad era ampliamente compartido entre los miembros del claustro. Una conversación en el pasillo o un momento de esparcimiento en común se convierten en valiosas oportunidades de aprendizaje informal para el profesorado novel (Piot et al., 2010). Pese a ello, es interesante destacar que la efectividad formativa de este tipo de prácticas podría verse notoriamente incrementada mediante la búsqueda de espacios y de tiempos que estimularan la reflexión práctica compartida. La posibilidad de pensar en y sobre la acción, de manera colaborativa, ayudaría al profesorado novel a reconstruir sus propias narrativas profesionales, especialmente, en momentos de contra- 
dicción y dificultades (Sales et al., 2017; Symeou \& Karagiorgi, 2018). En cuanto a las estrategias empleadas en el centro para estimular la formación colaborativa en interculturalidad, los participantes resaltaron, con especial énfasis, la organización flexible de los equipos docentes, práctica ampliamente utilizada para afrontar los retos que la diversidad cultural plantea en el entorno escolar (Biasutti et al., 2019). Por el contrario, el análisis de problemas inciertos y complejos y el trabajo en equipo con otros centros parecieron ser los métodos menos empleados por los miembros del claustro, pese a las múltiples ventajas que la coordinación con otras instituciones parece reportar para el aprendizaje docente en interculturalidad (Little et al., 2019).

En lo que respecta a las experiencias de formación entre colegas en interculturalidad, las voces de los participantes subrayaron, con especial ahínco, la falta de oportunidades de aprendizaje, tanto a nivel de centro como durante su capacitación inicial. Tales resultados vienen a alinearse con los hallazgos de Symeou y Karagiorgi (2018), quienes señalaron la inadecuación de los programas de formación para dotar al profesorado de las habilidades necesarias para el diálogo intercultural en las aulas. Esta escasa capacitación parecía ser contrarrestada mediante el aprendizaje informal con el resto de integrantes del claustro (Piot et al., 2010). Ahora bien, de sus discursos se deduce que este tipo de prácticas no eran especialmente valoradas como una vía para la mejora profesional, sino, más bien, como una estrategia para garantizar el correcto funcionamiento del centro. Esta percepción podría venir provocada, entre otras razones, por la equiparación del desarrollo profesional con las fórmulas tradicionales de formación, como por ejemplo los cursos, pese al aparente fracaso que estas iniciativas tienen para el aprendizaje docente en interculturalidad (Hajisoteriou et al., 2018).

Con relación a las decisiones que el centro había adoptado para promover la cultura colaborativa, el profesorado novel remarcó, de modo iterativo, aquellas actuaciones que buscan la colaboración con el entorno, sobre todo con las organizaciones asociativas y con las familias. Algunos estudios, como el de Peńalva y Leiva (2019) y el de Aguado-Odina et al. (2017), inciden también en la relevancia que la participación de los agentes comunitarios y del contexto familiar tiene para la transformación social y la construcción del aprendizaje dialógico intercultural. Sin embargo, desde la óptica de los participantes, esta colaboración representaba más un recurso para la adecuada organización institucional que una posibilidad de aprendizaje. Frente a ello, las reuniones de coordinación fueron las medidas de carácter interno que, según los docentes, el centro había implementado con más frecuencia para promover la cultura de colaboración. La validez de estos encuentros ha sido puesta de manifiesto por otros autores, como por ejemplo Ohi et al. (2019), sobre todo a la hora de responder a los conflictos interculturales en las aulas. No obstante, una vez más, este tipo de reuniones fueron vistas por el profesorado novel como una condición para la calidad de la enseñanza y no tanto como un procedimiento de aprendizaje profesional.

Con la realización del presente estudio, parece haber quedado fuera de toda duda la necesidad de incidir en el estímulo de la cultura colaborativa para que los docentes comprendan plenamente la valía de esta a la hora de adquirir y desarrollar las competencias interculturales. Los retos y desafíos que plantea la diversidad en las aulas no pueden ser abordados mediante estrategias improvisadas y azarosas, sino que se precisa de nuevos modelos que pongan el énfasis en la colaboración y en la reflexión conjunta sobre la propia práctica docente. Ahora bien, si es importante capacitar al profesorado en el enfoque colaborativo para el aprendizaje en interculturalidad, no lo es menos cuidar la forma en la que se hace, ya que con una perspectiva inadecuada se corre el riesgo de provocar el surgimiento de actitudes negativas y prejuiciosas entre los que recién inician su práctica profesional (Stahl et al., 2019). Parece, por tanto, que la construcción de una nueva escuela democrática e intercultural requiere del diseño de un nuevo paradigma de aprendizaje docente, en el que la colaboración, la reflexión y el diálogo entre las diferentes culturas y experiencias se conviertan en sus ejes transversales (Leiva, 2017). 
En este contexto, el proyecto de comunidades de aprendizaje viene a representar una de las opciones más pertinentes y apropiadas para la transformación de las prácticas docentes y el avance hacia un enfoque intercultural más participativo y solidario (Hajisoteriou et al., 2018). A través de la colaboración y la apertura que brindan, la institución escolar deja de ser únicamente un espacio para la enseñanza y se convierte en un lugar de aprendizaje, de encuentro y de diálogo para el profesorado y la sociedad en su conjunto (Aguado-Odina et al., 2017). Dentro de este marco conceptual, la propuesta de formación debe dirigirse, de manera específica, a la construcción de una cultura compartida y negociada, respondiendo así a las necesidades y demandas del profesorado novel y del entorno inmediato. Abrir espacios para compartir la práctica, la observación mutua y la planificación conjunta debe de convertirse en una actuación esencial dentro de la comunidad, puesto que a través de esta el profesorado principiante adquiere el conocimiento intercultural experto y perfecciona su ejercicio profesional. Ahora bien, para que este modelo de formación colaborativa en interculturalidad resulte eficaz y exitoso, debe nacer de las necesidades específicas del contexto y de las relaciones de este con el entorno, ajustándose, además, a la realidad concreta que el profesorado novel encuentra en el aula (Zeng \& Day, 2019). Del mismo modo, también será precisa la participación equilibrada de todos los integrantes de la comunidad, la democratización de la estructura organizativa de los centros, el compromiso de la administración escolar y el apoyo de las organizaciones sociales, ya que, en caso contrario, la transformación de la escuela en un ente vivo, abierto y sensible a todas las formas de ser, hacer y pensar la diversidad, corre un serio riesgo.

\section{Referencias}

Aguaded-Ramírez, E., Bartolomei-Torres, P., \& Angelidou, G. (2018). Analysis of a project conducted on unaccompanied refugees children (MERNAs). Journal of New Approaches in Educational Research, $7(2), 116-124$.

http://dx.doi.org/10.7821/naer.2018.7.256
Aguado-Odina, T., Mata-Benito, P., \& Gil-Jaurena, I. (2017). Mobilizing intercultural education for equity and social justice. Time to react against the intolerable: a proposal from Spain. Intercultural Education, 28(4), 408-423. https://doi.org/10.1080/14675986.2017.1333874

Alviar-Martin, T., \& Baildon, M. (2017). Deliberating values for global citizenship: a study of Singapore's social studies and Hong Kong's liberal studies curricula. En S. Choo, D. Sawch, A. Villanueva \& R. Vinz (Eds.). Educating for the $21^{\text {st }}$ century. Perspectives, policies and practices from around the world (pp. 73-92). Springer.

https://doi.org/10.1007/978-981-10-1673-8

Arnaiz-Sánchez, P., de Haro-Rodríguez, R., \& Maldonado Martínez, R. (2019). Barriers to student learning and participation in an inclusive school as perceived by future education professionals. Journal of New Approaches in Educational Research, 8(1), 1824. http://dx.doi.org/10.7821/naer.2019.1.321

Biasutti, M., Concina, E., \& Frate, S. (2019). Working in the classroom with migrant and refugee students: the practices and needs of Italian primary and middle school teachers. Pedagogy, Culture \& Society. https://doi.org/10.1080/14681366.2019.1611626

Chamberlin-Quinlisk, C. (2010). Cooperative learning as method and model in second language teacher education. Intercultural Education, 21(3), 243255. https://doi.org/10.1080/14675981003760432

Chiappe, A., de Samper, A., Wills, A. E., \& Restrepo, I. (2019). Rethinking $21^{\text {st }}$ century schools: the quest for lifelong learning ecosystems. Ensaio: Avaliação e Políticas Públicas em Educação.

http://dx.doi.org/10.1590/s0104-40362019002702138

Delgado-Algarra, E., Bernal-Bravo, C., \& López-Meneses, E. (2019). Multicultural competence and cosmopolitan citizenship in the Hispanic-Japanese context of higher education. Journal of New Approaches in Educational Research, 8(2), 166-183.

http://dx.doi.org/10.7821/naer.2019.7.425

Domínguez, M. C. (2006). Investigación y formación 
el profesorado en la sociedad intercultural. Universitas. Elo, S., \& Kyngäs, H. (2008). The qualitative content analysis process. Journal of Advanced Nursing, 62(1), 107-115.

http://dx.doi.org/10.1111/j.1365-2648.2007.04569.x

Escarbajal, A. (2010). La escuela inclusiva en una sociedad pluricultural y la importancia del trabajo colaborativo. Enseñanza \& Teaching: Revista Interuniversitaria de Didáctica 28(2), 161-179.

https://cutt.ly/RrcyXY6

Fernández-Díaz, E., Gutiérrez, P., \& Fernández, L. (2019). University-school scenarios and voices from classrooms. Rethinking collaboration within the framework of an interuniversity project. Journal of New Approaches in Educational Research, 8(2), 79-95. http://dx.doi.org/10.7821/naer.2019.7.372

Hajisoteriou, C., Karousiou, C., \& Angelides, P. (2018). INTERACT: building a virtual community of practice to enhance teachers' intercultural professional development. Educational Media International, 55(1), 15-33.

https://doi.org/10.1080/09523987.2018.1439709

Hsieh, H., \& Shannon, S. (2005). Three approaches to qualitative content analysis. Qualitative Health Research, 15(9), 1277-1288.

https://doi.org/10.1177/1049732305276687

Huber, G., \& Gürtler, L. (2013). AQUAD 7. Manual: The analysis of qualitative data. Ingeborg Huber Verlag. https://bit.ly/2FPxV9Y

Hyry-Beihammer, E. K., Jokikokko, K., \& Uitto, M. (2019). Emotions involved in encountering classroom diversity: beginning teachers' stories. British Educational Research Journal, 45(6), 1124-1139. https://doi.org/10.1002/berj.3554

Kelchtermans, G. (2019). Early career teachers and their need for support: thinking again. En A. Sullivan, B. Johnson, \& M. Simons (Eds.). Attracting and keeping the best teachers. Issues and opportunities (pp. 83-98). Springer.

https://doi.org/10.1007/978-981-13-8621-3 5
Leeman, Y., \& van Koeven, E. (2019). New immigrants. An incentive for intercultural education? Education Inquiry, 10(3), 189-207.

https://doi.org/10.1080/20004508.2018.1541675

Leiva, J. (2017). La escuela intercultural hoy: reflexiones y perspectivas pedagógicas. Revista Complutense de Educación, 28(1), 29-43.

http://dx.doi.org/10.5209/rev RCED.2017.v28.n1.48589

Little, S., Golledge, M., Agarwalla, H., Griffiths, B., \& McCamlie, D. (2019). Global teachers as global learners: intercultural teacher training in international settings. London Review of Education, 17(1), 3851. https://doi.org/10.18546/LRE.17.1.04

Means, A. (2019). Hypermodernity, automated uncertainty, and education policy trajectories. Critical Studies in Education.

https://doi.org/10.1080/17508487.2019.1632912

Ministerio de Fomento. (2011). Catálogo de barrios vulnerables. https://bit.ly/2YX5QGs

Morales, K., Sanhueza, S., Friz, M., \& Riquelme, P. (2017). The intercultural sensitivity of Chilean teachers serving an immigrant population in schools. Journal of New Approaches in Educational Research, 6(1), 71-77.

https://doi.org/10.7821/naer.2016.8.173

Ohi, S., O’Mara, J., Arber, R., Hartung, C., Shaw, G., \& Halse, C. (2019). Interrogating the promise of a whole-school approach to intercultural education: an Australian investigation. European Educational Research Journal, 18(2), 234-247.

https://doi.org/10.1177/1474904118796908

Pastor, E., \& Torralba, R. (2015). Trabajo social comunitario. Aprendiendo de las prácticas en barrios desfavorecidos de la Región de Murcia (España). Interacción y Perspectiva. Revista de Trabajo Social, 5(1), 12-35. https://bit.ly/39WZI6p

Peñalva, A., \& Leiva, J. J. (2019). Metodologías cooperativas y colaborativas en la formación del profesorado para la interculturalidad. Tendencias Pedagógicas, 33, 37-46.

http://dx.doi.org/10.15366/tp2019.33.003 
Piot, L., Kelchtermans, G., \& Ballet, K. (2010). Beginning teachers' job experiences in multi-ethnic schools. Teachers and Teaching, 16(2), 259-276. https://doi.org/10.1080/13540600903478524

Ponce, O., \& Pagán-Maldonado, N. (2015). Mixed methods research in education: capturing the complexity of the profession. International Journal of Educational Excellence, 1(1), 111-135. https://doi.org/10.18562/IJEE.2015.0005

Prendes, M., \& Román, M. (2017). Entornos personales de aprendizaje: una visión actual de cómo aprender con tecnologías. Octaedro. https://bit.ly/30p3noT

Sales, A., Moliner, L., \& Francisco, A. (2017). Collaborative professional development for distributed teacher leadership towards school change. School Leadership \& Management, 37(3), 254-266. https://doi.org/10.1080/13632434.2016.1209176

Savva, M. (2017). Learning to teach culturally and linguistically diverse students through cross-cultural experiences. Intercultural Education, 28(3), 269-282. https://doi.org/10.1080/14675986.2017.1333689

Stahl, G., Brock, C., Caldwell, J., Sharplin, E., \& Boyd, F. (2019). Dispositions towards diversity: two pre-service teachers' experiences of living and teaching in a remote indigenous community. Pedagogy, Culture \& Society. https://doi.org/10.1080/14681366.2019.1665090

Symeou, L., \& Karagiorgi, Y. (2018). Culturally aware but not yet ready to teach the "others". Reflections on a Roma education teacher training programme. Journal for Multicultural Education, 12(4), 314-329. https://doi.org/10.1108/JME-02-2017-0012

UNESCO (2015). Incheon Declaration. 2030 Education. Ensure inclusive and equitable quality education and promote lifelong learning opportunities for all. https://bit.ly/2PTesdi

Voss, T., \& Kunter, M. (2019). "Reality shock" of beginning teachers? Changes in teacher candidates' emotional exhaustion and constructivist-oriented beliefs. Journal of Teacher Education, 1-15. https://doi.org/10.1177/0022487119839700

Zeng, Y., \& Day, C. (2019). Collaborative teacher professional development in schools in England (UK) and Shanghai (China): cultures, contexts and tensions. Teachers and Teaching, 25(3), 379-397. https://doi.org/10.1080/13540602.2019.1593822

\section{CÓMO CITAR:}

Roig-Vila, R., \& Urrea-Solano, M. E. (2020). Formación colaborativa en interculturalidad del profesorado de Educación Infantil y Primaria. Revista Caribeña de Investigación Educativa (RECIE), 4(1), 7-19. https://doi.org/10.32541/recie.2020. v4i1.pp7-19 